\title{
Depiction of Lying Down and Standing up Sequences in Multiparous Sows
}

\author{
A.S. Leaflet R3198 \\ Jared Mumm, Graduate Research Assistant; \\ Joseph Stock, Graduate Research Assistant; \\ Samaneh Arzapajouh, Postdoctoral Research Associate; \\ Amber Howell, Undergraduate Research Assistant; \\ Anna K. Johnson, Associate Professor; \\ Kenneth J. Stalder, Professor, Department of Animal \\ Science, Iowa State University; \\ Julia A. Calderón Díaz, Postdoctoral Research Fellow, Pig \\ Development, Teagasc Moorepark Grassland Research and \\ Innovation Centre
}

\section{Summary and Implications}

The objective of this study was to create a pictorial lying down-standing up sequence depiction in multiparous sows. Eighty-five multiparous sows were moved from their home stall to a testing stall where they were video recorded for one lying down-standing up event on 30,60 and 90 days of gestation. The digital video camera was positioned on the adjacent stall so the sows' profile was visible while recording. Observations ceased when the sow successfully lied down or if 2.5 hours elapsed since recording began. Normal standing and lying pictorial depictions were created, and deviations from the normal lying down and standing up sequences were also pictorially depicted. This is the first published pictorial depictions on mulitparous sows on the standing-lying-standing sequence.

\section{Introduction}

The pig lying down and standing up sequence was first described by Baxter and Schwaller (1983). These authors suggested that locomotory problems would cause few behavioral alterations during this sequence. Since the early 1980's the U.S. swine industry has experienced rapid genetic improvements which may have influenced how pigs position their bodies while transitioning from standing to lying or vice versa. To our knowledge, there are no other studies that describe and provide a pictorial depiction of the rising and lying down postural sequences in multiparous sows. Therefore, the objective of this study was to create a pictorial lying down-standing up sequence depiction in multiparous sows.

\section{Materials and Methods}

The protocol for this work was approved by the ISUIACUC committee. This work was conducted from August 2015 to June 2016.
Animals: Eighty-five multiparous crossbred sows (parities 1 to 4), were moved from their home stall to a testing stall on 30,60 and 90 days of gestation.

Behavioral equipment: A digital video camera (GoPro Hero, GoPro Inc., San Mateo, CA, USA) was positioned on the adjacent stall by a camera clamp, approximately $51 \mathrm{~cm}$ from the floor.

Digital video recording: The video provided a continuous sow side profile. Observations ceased when the sow successfully laid down (defined as the sow lying in either sternal or lateral recumbency not supported by any of her legs) and stood up or if 2.5 hours elapsed.

Sample Points: Video was continuously viewed, and sow postures and movements that occurred during the lyingstanding sequence were identified. A behavioral sampling methodology focused on attempted and a successful lying and standing sequence was used. Lying; 1) the sow drops to a knee, 2) she rotates her shoulder and head to rest them flat on the ground and 3) lowers her hindquarters to the ground. Standing; 1 ) the sow extends her front feet from under her body, 2) she rises to a sitting position bearing weight on her front feet with hindquarters resting on the ground and 3) raises her hindquarters off the ground to bear weight on all four feet.

Creation of pictorial lying down-standing up sequence depiction: Still frames were cut from the video using AVcutty v3 (Andreas von Damaros, Krefeld, Germany). The still images were then traced by hand on carbon paper and scanned in sequence to achieve the pictorial lying down-standing up sequence depictions.

\section{Results and Discussion}

Depictions of a normal lying sequence can be visualized in Figure 1A. The most common deviations were; sows did not lay down in the allocated observation time (i.e. 2.5 hours), sows did not rotate their shoulder, or sows finished the lying down sequence in the sitting position (Figures 1B-E). Depictions of a normal standing sequence can be visualized in Figure 2A. The most common deviations for the standing sequence was the sow skipped the sitting posture and simply rose straight up from the lying position (Figures 2B-E).

\section{Acknowledgements}

This project was funded by the National Pork Board grant \#15-004. We would like to thank the staff at the Lauren Christian Swine Research Center for all their help during this study. 
Figure 1. Different postural combinations observed during the lying down sequence. A) normal lying down sequence, B to $E$ represent deviations from the normal lying down sequence

A

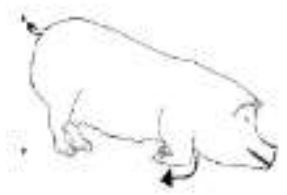

i) Kneeling

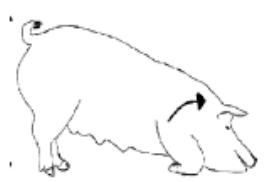

ii) Shoulder rotation

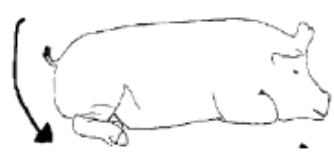

iii) Lower hindquarters and sow lands on floor

B

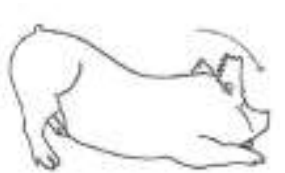

i) Bow

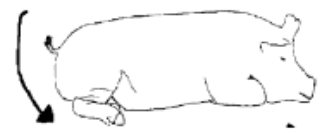

ii) Lower hindquarters and sow lands on floor

C

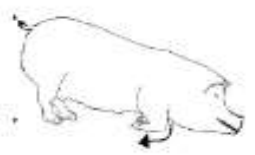

i) Kneeling

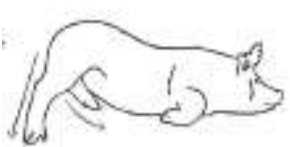

ii) Back legs slip

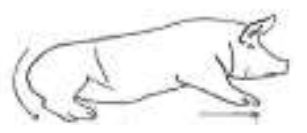

iii) Lower hindquarters and stretch front legs

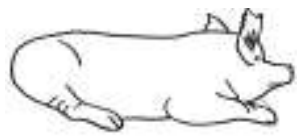

iv) Sow lands on floor
D

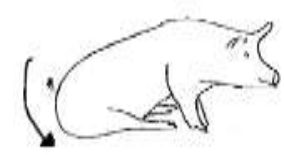

iii) Lower hindquarters

$\mathbf{E}$

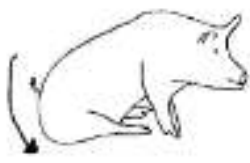

iii) Lower

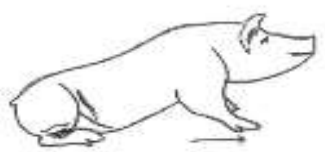

ii) Bow

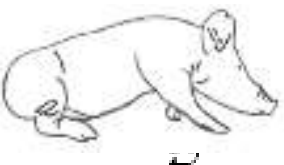

ii) Kneeling

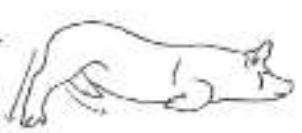

iii) Sow lands on floor

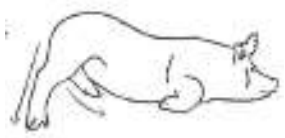

iii) Sow lands on floor 
Figure 2. Different postural changes documented during the standing up sequence. A) normal standing sequence, B to D represent deviations from the normal sequence.

A

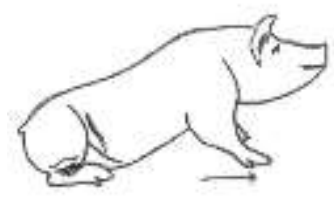

i) Legs folded beneath body

B

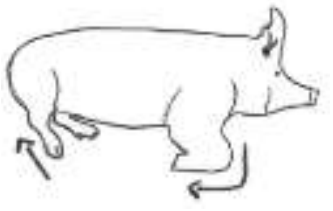

i) Sow 'kneels' and starts to lift hindquarters

C

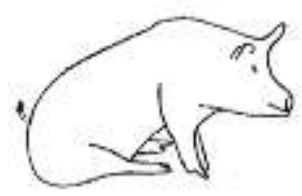

i) Sow rises to a sitting position

D

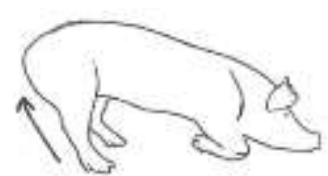

i) Lift hindquarters

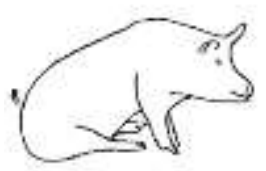

ii) Sow rises to a sitting position

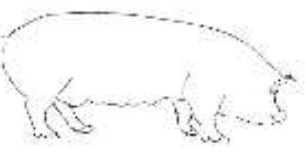

iii) Lift hindquarters and sow achieves a full standing

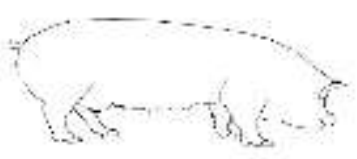

ii) Sow achieves a full standing position

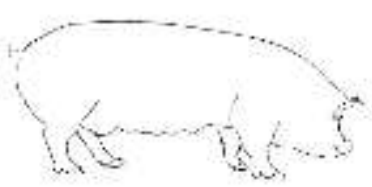

ii) Lift hindquarters and sow achieves a full standing position

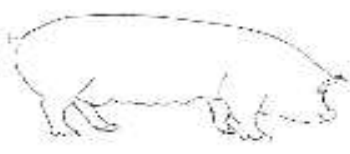

ii) Sow achieves a full standing position 